\title{
Impacto académico y profesional de un programa de educación física a nivel universitario Academic and professional impact of university physical education program
}

\author{
Jayson Bernate*, Ingrid Fonseca*, Milthon Betancourt*, Alexander Guataquira**, Johnatan Rodríguez***, Miguel \\ Rodríguez*** \\ *Corporacion Universitaria Minuto de Dios, **Secretaria de Educación Distrital, ***Semillero de Investigación Calidad Educativa
}

\begin{abstract}
Resumen. Este artículo tuvo como objetivo medir el impacto académico y profesional de la licenciatura en educación física de la Corporación Universitaria Minuto de Dios UNIMINUTO sede principal. Para ello se aplicó el test de Evaluación del impacto académico en programas de educación superior, elaborado por Gómez, Quiroz \& Zambrano (2016) compuesta por 3 variables y 32 ítems. La muestra se aplicó a 150 estudiantes de octavo y noveno semestre y la metodología es de carácter cuantitativo no experimental y alcance descriptivo. Para el estudio se utilizó el software SPSS, el análisis estadístico se realizó con técnicas de estadística descriptiva y las variables analizadas fueron: grupo I: aprendizaje, grupo II: satisfacción personal, grupo III: desempeño profesional. Los principales hallazgos reflejan que el aprendizaje, la satisfacción personal y el desempeño profesional, arrojan puntuaciones aceptables respecto a la media aritmética. Se concluye que el programa académico evaluado aporta significativamente a la formación integral de los educadores físicos, en cuanto a habilidades profesionales propias del área específica y desarrolla competencias sociales, en pro de innovar continuamente la capacitación docente en educación superior.
\end{abstract}

Palabras clave: impacto, educación física, programa académico, profesional.

\begin{abstract}
This article aimed to measure the academic and professional impact of the bachelor's degree in physical education at the Minuto de Dios UNIMINUTO University Corporation headquarters. For this, the test of Evaluation of the academic impact in higher education programs was applied, prepared by Gómez, Quiroz \& Zambrano (2016) composed of 3 variables and 32 items. The sample was applied to 150 eighth and ninth semester students and the methodology is quantitative, not experimental, and descriptive in scope. The SPSS software was used for the study, the statistical analysis was carried out with descriptive statistics techniques and the variables analyzed were: group I: learning, group II: personal satisfaction, group III: professional performance. The main findings reflect that learning, personal satisfaction and professional performance yield acceptable scores with respect to the arithmetic mean. It is concluded that the evaluated academic program contributes significantly to the comprehensive training of physical educators, in terms of professional skills specific to the specific area and develops social skills, in order to continuously innovate teacher training in higher education.
\end{abstract} Keywords: impact, physical education, academic, professional program.

\section{Introducción}

Históricamente los programas universitarios impactan de manera académica y profesional a sus estudiantes, desde hace un tiempo se utilizan diferentes instrumentos para medir y evaluar dicho impacto, esto debido a que los entes gubernamentales como lo son; El Ministerio de Educación (MEN) y las secretarias departamentales encargadas de velar por el buen desarrollo de la educación, crean y formulan leyes y decretos de calidad educativa, los cuales buscan mejorar cada día los programas académicos en la educación superior. En este caso se medirá el impacto académico y profesional de un programa de Licenciatura en Educación Física.

Es importante medir el impacto de los programas en las universidades de Colombia, para ver de qué manera le aportan a la sociedad, y observar si los estudiantes de cada universidad se sienten capacitados al momento de poner en práctica lo aprendido y si hay falencias para ver de qué manera se podría solucionar cada problema, ya sea de tipo social o del área específica.

En tal sentido, Roca et al. (2017), sugieren estudiar el comportamiento de respuesta del estudiante ante variables cognitivas y afectivas para determinar la intención de recomendar el servicio de formación. La razón de esa investigación responde a una falta de estudios en el sector en referencia a los componentes actitudinales e intencionales

\footnotetext{
Fecha recepción: 13-06-20. Fecha de aceptación: 15-08-20 Jayson Bernate
}

jayson.bernate@uniminuto.edu de los estudiantes, así como el efecto moderador que el rendimiento académico o la calidad tangible de la educación superior, pueden tener en la satisfacción y en la intención de recomendación.

En la misma línea, Zueck et al. (2019), proponen una metodología de 10 pasos para la medición del impacto estudiantil en instituciones de educación superior. Esta metodología con su enfoque sistemático, presenta carácter dinámico, lo que permite su adaptación a otros entornos. Además, constituye un aporte para que las instituciones de educación superior puedan mejorar continuamente, con la intención de lograr una mejor calidad educativa, puesto que el estudiante sería un cliente satisfecho.

La calidad es un elemento clave para que una organización sea competitiva. Sin embargo, al ser una característica dinámica y específica para cada sector, es necesario contar con las herramientas adecuadas para evaluarla. En específico, las universidades necesitan un sistema de evaluación y acreditación que les permita participar activamente en el proceso de globalización académica (Campos et al., 2015).

Además, al momento de aplicar los modelos de calidad educativa se pueden identificar varios inconvenientes o problemáticas, respecto al entorno del aprendizaje en los estudiantes, como en la enseñanza de los docentes (Paéz \& Hurtado, 2018). A pesar de las mejoras a nivel gubernamental en contratación y capacitación docente, aún se encuentran lejos de lo ideal los presupuestos asignados para la educación, como es el caso de Colombia, donde anualmente estos son mínimos para apuntarle a una revolución de la calidad en educación.

Igualmente se puede dar referencia a las metodologías 
que se utilizan en las aulas de clase, y cómo estas influyen en los estudiantes, de manera positiva o negativa. Puesto que ello, es un factor determinante en la percepción del estudiantado quienes advierten cuando un docente tiene un alto o bajo nivel de apropiación de las temáticas y conceptos a trabajar en la clase (Garcia et al, 2020). Es decir, que si el educador presenta falencias en el aula de clase, frente al dominio de temáticas o aplicación de estrategias esto podría llevar a la insatisfacción en los procesos de formación del educando.

Por otro lado, es claro que las condiciones de calidad que rige un programa de educación superior, están sucedidos de múltiples factores, en esta investigación se abordarán los siguientes: aprendizaje, satisfacción personal y desempeño profesional.

Por tal motivo, el aprendizaje en la educación superior influye a nivel social interviniendo un quehacer para el bien de la comunidad, en tal sentido Serra et al. (2016), plantean que:

El proceso de formación universitaria supone un constructo complejo para los diferentes actores del sistema educativo. En el caso de directivos y profesores, cómo adaptar el currículum a las necesidades y características de los estudiantes, sigue siendo un determinante fundamental en la construcción de procesos de enseñanza-aprendizaje de calidad. (p. 62)

A nivel social se deben entender ciertos aspectos, que influyen en el estudiante en formación docente, como la familia, amigos o compañeros de universidad y trabajo, el contexto donde vive, situaciones donde labora o prácticas profesionales y relación académica con la universidad, aquello conlleva a que el estudiante aproveche el aprendizaje en su tiempo académico o libre, para fortalecer las habilidades docentes.

De igual forma, Fernández \& Méndez (2015), sugieren que un modelo de enseñanza;

Plantea una visión de esta que considera a la vez las teorías del aprendizaje, los objetivos de aprendizaje a largo plazo, el contexto, el contenido, la gestión del aula, las teorías de enseñanza, el control del proceso y la evaluación del aprendizaje del estudiante (p. 201).

Medir el impacto del programa en educación física, del rol docente UNIMINUTO, sirve para generar acciones de cambio mediante las prácticas docentes a través del enfoque praxeológico el cual se define como «un proceso reflexivo que parte del análisis crítico de las prácticas y experiencias de cada persona o comunidad» como lo afirma (Juliao, 2011, p. 2).

La formación en licenciatura de educación física, aporta conocimientos de enseñanza-aprendizaje, para saber y entender cómo socializa el hombre en comunidad, interactuando como ser axiológico, respondiendo a las necesidades sociales de los diferentes entornos educativos dentro y fuera del aula así como «a la necesidad del país de tener personas versátiles, creativas y con capacidad de innovación, abiertas a nuevos aprendizajes y dispuestas a trabajar en equipo» (Bernate, Bejarano \& Cardozo, 2020, p.559), por ende la educación física, trabaja de la mano, articulando diferentes campos interdisciplinares y gestionando proyectos de impacto social, de tal manera que busque diferentes puntos de vista para encontrar soluciones a la comunidad, por lo anterior los licenciados en educación física, interactúan de forma socio crítica en el ámbito escolar y cultural.

Son varias las causas que pueden interferir significativamente en el aprendizaje de los estudiantes universitarios. Por tal motivo debe encontrar estrategias para saber de qué forma puede aprender mejor. En este sentido Castro et al. (2015), plantean que «las estrategias de aprendizaje se entienden como un conjunto de procesos que pueden facilitar la adquisición, almacenamiento y utilización de la información» (p.5). Es decir, se busca la forma interactiva para que los estudiantes presten más atención al momento de recibir la clase y generen una adquisición de conocimiento y lo puedan poner en práctica.

De otra manera, es clave precisar en la educación superior la importancia de tener en cuenta, que tan satisfechos se sentirán los estudiantes al haber culminado su carrera universitaria, y sí se cumplieron las expectativas al desarrollar el programa mencionado, con lo cual se permite enfocar a su nivel de competencia profesional, académica, personal y social. Es así como Surdez et al. (2018), identificaron las áreas de oportunidad para incrementar la satisfacción estudiantil en referencia a infraestructura y retroalimentación positiva del trato respetuoso que reciben los estudiantes de sus tutores y a las oportunidades que les brinda la institución para su desarrollo personal. Así mismo, especifica diferencias de satisfacción estadísticamente significativas entre grupos de edad, campus al que pertenece el estudiante, programas de estudio y estado civil.

Varias dificultades y problemáticas se presentan, entre ellas el diseño curricular, infraestructura deficiente, falta de capacitación docente para mantener una actualización, creatividad y desempeño en las clases, falta de materiales didácticos, laboratorios, recursos digitales. Fonseca et al. (2019)

Afectando de manera significativa la calidad en los programas de educación superior, y considerando la administración de la calidad total, se basa en que los usuarios sean servidos al máximo grado posible, para que los servicios y productos satisfagan sus requerimientos y necesidades. El usuario juzga si la calidad de los bienes y servicios es aceptable y satisface sus necesidades y es él, quien debe fungir como centro de la organización que busca la excelencia (Clemenza, Ferrer y Pelekais, 2005).

En el contexto latinoamericano Cruz, Toro \& Duarte (2009), destacan el carácter polisémico del concepto de calidad y los retos que plantea a la educación superior, en su devenir cotidiano, asumir una perspectiva de la calidad ligada tanto a las demandas de los contextos educativos reales, como a las expectativas de los diferentes actores sociales, que cohabitan por la institucionalidad educativa y la concreción de una perspectiva de derechos en las prácticas escolares, que potencie la subjetividad política, en particular de los estudiantes universitarios. Por ende, el estudiante universitario próximo a graduarse, debe tener cualidades y capacidades para dar soluciones a problemáticas y dificultades, que se presenten en su futuro profesional. Bernate et al. (2019)

También la importancia del desempeño profesional está 
siempre presente en cuanto al ámbito pedagógico y laboral ya que se puede tener en cuenta la calidad y el rendimiento de los docentes de educación física en el proceso que lleva desarrollando, así garantizar su idoneidad docente. Dando una aproximación en cuanto a egresados. Bernate et al. (2019)

Además, cuando se reflexiona en un contexto social de calidad educativa se debe tener un adecuado desempeño profesional para ejercer un seguimiento o procesos fundamentales en el aprendizaje de los estudiantes se procede las competencias laborales. Urrea et al. (2019)

La educación física tiene varios ámbitos para desempeñar laboralmente, para ello se quiere diferentes acciones y formas de actuar como docente, Urrea et al. (2018) «algunos de los requerimientos específicos de los nuevos profesionales para encaminarlos hacia la gestación de nuevos saberes que posibiliten cambios e innovación continua en los contextos laborales en los que se encuentren inmersos.» (p. 3).

En cuanto a la formación en los programas de Licenciatura en Educación Física el desempeño profesional en la calidad educativa genera un alto impacto social, el aprendizaje y exigencias del mismo en un contexto educativo, social, profesional, laboral. Bernate et al. (2020) En las prácticas profesionales es importante determinar si se lleva a cabo un óptimo desempeño profesional y si este en las clases de aula o de campo, utilizan las metodologías e instrumentos de enseñanza y aprendizaje de forma apropiada y eficaz, frente a las competencias generales y específicas del ser docente, Además de cuanto los estudiantes aplican y transfieren la filosofía de la universidad de donde se enriquecieron cognitivamente durante toda su formación profesional y académica, es decir si el acompañamiento de los docentes de prácticas profesionales es suficiente para cumplir expectativas de aprendizaje y el desarrollo de un proyecto educativo para la comunidad, varios aspectos claves que encamina al estudiante próximo graduado para insertarse profesionalmente al mundo laboral.

El estudiante licenciado en formación debe ser realista en sus acciones pedagógicas, crear y desenvolver su método de enseñanza para las futuras generaciones, dar lugar a la comunidad para debatir y discutir ideas para generar espacios de interacción social, identificar el estilo de enseñanza y aprendizaje para hacer llegar a la comunidad lo indispensable de la educación física en las aulas y campos deportivos, las actividades recreativas de relación interpersonal e intrapersonal y el deporte como ente socializador, desarrollo de habilidades cognitivas, para así acrecer la aptitud y actitud en sus quehaceres y reflexionar sobre el estado de ánimo en solventar las problemáticas presentes, aumentando los niveles de habilidades motrices y sociales en determinada comunidad educativa especifica. Bernate et al. (2019) \& Rojas et al. (2019)

Para finalizar este apartado cabe citar los antecedentes investigativos en torno a la medición del impacto en programas de educación superior; por ello Castrillón \& Giraldo (2016) recomiendan que para hacer efectiva una medición de este tipo se debe realizar una caracterización de los egresados, una parametrización sobre impacto en el medio, un modelo de gestión de egresados y unas recomendaciones para el sistema de información para el seguimiento a los egresados.
Por otra parte, Tejada Fernández \& Fernández La Fuente (2012) Citado por Gracia (2018) determinaron que el proceso de evaluación debe realizarse diferida a la finalización del programa académico, con la finalidad de eliminar posibles errores derivados de las implicaciones de los participantes en el hecho formativo y verificar el impacto pasado un tiempo después de la acción formativa, esta evaluación se lleva a cabo con el fin de verificar la permanencia y consistencia de los cambios producidos en los sujetos, la mejora de las prácticas profesionales, los cambios institucionales, etc., según las metas del plan de formación.

Por último, Maya \& Herrera (2011) en su investigación se concentraron en indagar aspectos generales y particulares relacionados con la población de graduados de los programas de Licenciatura en Educación Básica, Licenciatura en Filosofía y Educación Religiosa, Ingeniería Informática, Psicología y el programa de posgrado Especialización en Pedagogía de la Virtualidad. La indagación permitió obtener resultados de variación en aspectos de tipo demográfico, laboral y académico de los graduados, así como la percepción de satisfacción con el programa respectivo y los servicios de la Institución por parte de graduados y empleadores.

De acuerdo a lo anteriormente socializado el objetivo de esta investigación es medir el impacto académico y profesional en la educación del programa universitario Licenciatura en Educación Física de la Corporación Universitaria Minuto de Dios UNIMINUTO, sede principal modalidad presencial.

\section{Metodología}

\section{Participantes}

La muestra de esta investigación estuvo constituida por incidencia de 150 estudiantes de los semestres $8^{\circ}$ y $9^{\circ} ; 108$ son de género masculino $(72 \%)$ y 42 de género femenino $(28 \%)$ con el siguiente rango de edad (15-19) 8 personas $(5,3 \%),(20-25) 133$ personas $(88,6 \%),(26-30) 9$ personas $(6$ $\%)$. Con relación al estrato socioeconómico, la población en su mayoría es de estrato 3 (79 personas) (52,6\%), seguido del estrato 2 (66 personas) (44\%), estrato 1 (4 personas) $(2,6 \%)$ y estrato 4 ( 1 persona) $(0,6 \%)$. Los estudiantes que hicieron parte de esta investigación pertenecen a la Corporacion Universitaria Minuto de Dios UNIMINUTO, Sede Principal de la localidad de Engativá, Bogotá Colombia.

\section{Diseño}

El diseño empleado en la presente investigación ha sido un cuantitativo, no experimental, tipo encuesta y trasversal. Se ha optado por este tipo de enfoque metodológico debido a que se trata de un método de investigación capaz de dar respuesta a problemas tanto en términos descriptivos como de relación de variables cuando la información es recogida de forma sistemática, garantizando el rigor de los datos obtenidos. Tal y como señalan Hernández-Pina y Maquilón (2010) los diseños mediante encuesta son muy habituales en el ámbito de la educación ya que son aplicables a múltiples problemas y permiten recoger información sobre un número elevado de variables.

\section{Instrumento}

El instrumento utilizado fue el test de Evaluación del 
impacto académico en programas de educación superior, elaborado y validado por Gómez, Quiroz \& Zambrano (2016) compuesto por 3 variables y 32 ítems, distribuidos de la siguiente manera, variable 1: satisfacción personal con 12 ítems, variable 2: aprendizaje con 14 ítems y variable 3: desempeño profesional con 6 ítems. El formato de respuesta fue por medio de una escala tipo Likert con 5 opciones de respuesta: 1 (nada) 2 (poco) 3 (algo) 4 (bastante) 5 (mucho).

\section{Procedimiento}

Para la recolección de los datos, se informó a los participantes sobre la naturaleza de la investigación, se pidió la colaboración de las directivas de la Institución Universitaria, los docentes y los estudiantes. Se entregó un formato de autorización dirigido a los estudiantes universitarios, solicitando su consentimiento para la participación en el estudio. El día de la aplicación del cuestionario se explicó el objetivo del mismo y las variables. Cada estudiante tuvo 40 minutos para contestar de forma auto administrada.

El análisis estadístico se realizó por medio del programa SPSS 23.0, se hizo un análisis de la fiabilidad del instrumento, se calcularon los estadísticos descriptivos de las variables y dimensiones del estudio teniendo en cuenta la media aritmética y desviación típica, para posteriormente realizar la discusión y extraer las conclusiones del estudio.

\section{Resultados}

Con la finalidad de hacer un análisis de la fiabilidad del test aplicado a los 242 estudiantes se evidencia una fiabilidad adecuada de la escala total (,901). Lo cual determina que la información recogida es confiable ya que se demuestra en la validación del instrumento utilizado en la investigación, basado en la resultante superior de confiabilidad al 0,7, lo cual significa que la información suministrada es confiable para la investigación.

\begin{tabular}{|c|c|c|}
\hline Variable & Alpha de Cronbach & Número de Elementos \\
\hline Satisfacción Personal & 867 & 12 \\
\hline Aprendizaje & ,987 & 14 \\
\hline Desempeño Profesional & 849 & 6 \\
\hline Escala Total & ,901 & 32 \\
\hline \multicolumn{3}{|c|}{$\begin{array}{l}\text { Tabla } 2 . \\
\text { Estadística Descriptiva de las Variables }\end{array}$} \\
\hline Variable & Media Aritmética & Desviación Típica \\
\hline Satisfacción Personal & 4,3 & 0,26 \\
\hline Aprendizaje & 4,1 & 0,25 \\
\hline Desempeño Profesional & 4,2 & 0,27 \\
\hline Promedio General & 4,1 & 0,25 \\
\hline \multicolumn{3}{|c|}{$\begin{array}{l}\text { Tabla } 3 . \\
\text { Estadística Descriptiva de la repercusión en la población estudiantil }\end{array}$} \\
\hline Variable & \multirow{2}{*}{\multicolumn{2}{|c|}{ Promedio }} \\
\hline Satisfacción personal & & \\
\hline Aprendizaje & \multicolumn{2}{|r|}{$\begin{array}{l}14,8 \\
22,2\end{array}$} \\
\hline Desempeño profesional & \\
\hline Promedio total & \multicolumn{2}{|r|}{$\begin{array}{l}38,0 \\
75,0\end{array}$} \\
\hline \multicolumn{3}{|l|}{$\begin{array}{l}\text { Tabla } 4 . \\
\text { Correlaciones entre Variables }\end{array}$} \\
\hline \multicolumn{3}{|l|}{ Variable } \\
\hline \multirow[t]{3}{*}{ Aprendizaje } & Correlación de 1 Pearson &, $517^{* *}$ \\
\hline & Sig. (bilateral) & 0,000 \\
\hline & N 150 & 150 \\
\hline \multirow{4}{*}{ Desempeño Profesional } & Correlación de 1 Pearson &, $517 * *$ \\
\hline & Sig. (bilateral) & 0,000 \\
\hline & & 1 \\
\hline & N 150 & 150 \\
\hline \multicolumn{3}{|l|}{$\begin{array}{l}\text { Tabla } 5 . \\
\text { Resumen del Modelo }\end{array}$} \\
\hline Modelo & R cuadrado & $\mathrm{R}$ cuadrado ajustado \\
\hline, $517^{\mathrm{a}}$ & 0,267 & 0,262 \\
\hline
\end{tabular}

Los resultados sobre la evaluación en el impacto académico del programa universitario investigado muestran un índice de satisfacción alto discriminado según la escala (Nada, Poco, Algo, Bastante, Mucho), frente a los objetivos planteados al inicio del programa por parte del estudiante, al igual que su cumplimiento durante el desarrollo de la carrera. Se acentúa en las necesidades de formación y las expectativas presentadas por la facultad. Con una media aritmética general de 4,1 y una desviación típica de 0,25 se puede observar que la mayoría de los promedios presentados en la tabla anterior se encuentran sobre 4, mostrando estar bastante satisfechos en las variables de satisfacción personal, aprendizaje y desempeño profesional de aprendizaje.

La encuesta se estructura en tres aspectos los cuales están valorados $20 \%, 30 \%$ y $50 \%$ respectivamente. Teniendo en cuenta una estimación del $75 \%$ en el puntaje global permite determinar la repercusión que tiene en la población estudiantil la calidad en la formación presente en este programa y su proyección dentro de las otras facultades

Encontrando que la relación es positiva pero no es tan fuerte $(0,51)$ sobre estos dos aspectos, y están relacionados según el $\mathrm{R}^{2}$ en un $26 \%$ como lo muestra la siguiente tabla de relación.

\section{Discusión}

Por medio del instrumento se evaluó el impacto del programa académico teniendo en cuenta la relación entre las actividades planificadas y realizadas. El cuál estuvo compuesto de 3 variables, la primer variable fue satisfacción cuyas categorías fueron cumplimiento de los objetivos del docente y del programa académico, la segunda variable fue el aprendizaje y las categorías fueron conocimientos adquiridos y habilidades y la última fue desempeño cuyas categorías fueron destreza para el trabajo y actitud hacia el mismo.

Teniendo en cuenta los resultados de la encuesta, la puntuación en general tiene un promedio alto con relación al impacto del programa académico, se observa que la satisfacción personal obtiene una puntuación más alta frente a las variables de aprendizaje y desempeño profesional. Estos resultados se relacionan con la investigación realizada por Pérez-Albéniz, Pascual, Navarro, \& Lucas-Molina (2015), quienes analizaron el impacto de un programa universitario en España y plantean que los estudiantes se encuentran satisfechos a nivel académico, social, cognitivo, físico y psicológico.

A continuación, se discute el análisis de los resultados acerca del impacto académico y profesional de la Licenciatura de educación física, recreación y deportes. En este sentido, cabe señalar en primera instancia que las universidades son espacios en los cuales se debe promover la formación integral de los estudiantes a nivel profesional, social y personal, para lo cual es necesario fomentar que los mismos desarrollen estrategias para poder desempeñarse en sus actividades académicas (Hernández, Lópezy Olmedo, 2015), de esta forma se crean espacios para que los nuevos licenciados fortalezcan los aprendizajes obtenidos y pongan en práctica los conocimientos adquiridos en el transcurso de la carrera y de este modo desarrollar nuevas habilidades. 
También se debe tener en cuenta la motivación, ya que se incentiva el logro del objetivo o la meta planteada, por tanto, es más un proceso que un producto e implica la existencia de unas metas a corto, mediano y largo plazo. Se debe fortalecer la motivación intrínseca y extrínseca ya que durante el proceso educativo se pueden presentar diversos obstáculos, que influyen en la pérdida del interés de los estudiantes y en consecuencia bajos resultados de aprendizaje, por lo tanto, se debe promover la capacidad de sobrellevar las presiones académicas y sociales para asumir compromisos y desafíos. (Ryan \& Deci, 2000; Gallardo \& Carter, 2015).

De la misma forma, es pertinente acotar que durante el proceso de formación en los licenciados en educación física, es muy importante evaluar durante la carrera si el estudiante ha dado cumplimiento con los objetivos de aprendizaje, entrelazando la relación directa de programa-estudiante y en coherencia con los resultados, se podrá parametrizar el impacto del programa académico en cuestión. Tal como lo plantea la UNESCO(1998),

los estudiantes y sus necesidades deben situarse en el centro de las preocupaciones de los responsables de la adopción de decisiones en el plano nacional e institucional, de manera que se constituyan en participantes esenciales y protagonistas en el proceso de renovación de la educación superior, especialmente en lo que atañe a la enseñanza, la evaluación, la renovación de los métodos pedagógicos, así como de su participación en la elaboración de las políticas institucionales y en la gestión de los establecimientos. (p. 20)

Esto quiere decir que el estudiante debe ser parte fundamental en la renovación pedagógica de las instituciones, ya que al sentirse partícipe de ello se siente importante e involucrado en los procesos que se lleven a cabo y de esta forma lo ayuda a sentirse motivado y ser autónomo de sus propias decisiones. Las universidades deben atender las necesidades de los estudiantes, en donde se pueda tener en cuenta lo que ellos en realidad quieren y necesitan aprender, ya que esto los beneficia en su vida personal y de ahí depende la permanencia de los mismos y el grado de satisfacción. Así como lo mencionan Álvarez et al. (2015), «para el perfil del egresado se hace indispensable capacitar a los estudiantes para que pongan de manifiesto los objetivos, fundamentos, conceptos esenciales de la materia y los métodos, medios y procedimientos del contenido a desarrollar.» (p.4)Así mismo, cobra importancia que el estudiante mida sus habilidades y conocimientos para planificar y ejecutar clases teóricas y prácticas en todo tipo de población, teniendo presente la diversidad, en donde la persona sienta que en verdad aprende algo para la vida.

Es así como los docentes de UNIMINUTO deben presentar contenidos en sus clases para promover la dimensión social, como un método para crear espacios de discusión y debate (Bernate et al, 2019). Con relación al rol del docente, Callado (2015), considera que deben estar en capacidad de observar las formas en las que los estudiantes aprenden e involucrar diferentes metodologías, teniendo en cuenta la necesidad de implementar nuevas didácticas para que todos aprendan y sean evaluados en relación a las competencias a desarrollar, en la construcción de las capacidades del ser docente para adquirir nuevas habilidades y conocimientos.

La variable desempeño profesional obtuvo un buen puntaje por parte de los estudiantes, en este sentido se resalta que la universidad que oferta el programa objeto de estudio se fundamenta en una educación de equidad para gestionar oportunidades de participación. En este orden de ideas Arciniegas \& Mejia (2017), consideran que «tanto la concepción o planificación de la clase como la dirección del proceso con los alumnos, requiere del profesor una alta maestría pedagógica, para conducir adecuadamente el aprendizaje de sus educandos» $(\mathrm{p}, 44)$. Por ende, se promueven los aprendizajes para el desarrollo de destrezas para el trabajo por medio de la buena planificación de clases y la creación de espacios para la participación en contextos sociales en el desarrollo de un currículo pertinente y acertado en las funciones del docente, el cual desempeñe habilidades que le favorezcan en tareas de la vida laboral.

Otro aspecto a tener en cuenta son los estilos de enseñanza y aprendizaje, los primeros se calculan a partir del entorno social y educativo, es decir, teniendo en cuenta la edad, la economía, aspectos sociales, condiciones educativas, recursos humanos y físicos, el desempeño profesional a partir de los acontecimientos vividos y los principios y fundamentos de la educación física. Con relación a los segundos, Betancourt et al. (2020) señalan que: «durante el transcurso de una carrera universitaria los estilos de aprendizaje se modifican. Así, la predominancia o tendencia de ciertos estilos se vería alterada durante los últimos semestres y periodos de finalización» (p.63), esto sucede debido al tipo de metodología desarrollada por los docentes y la acomodación del aprendiz a los nuevos contenidos y conocimientos a desarrollar, y su habilidad para adaptarse al contexto.

De la misma forma, Clereci \& Da Re (2019), recomiendan realizar tutorías para lograr mejorías en el proceso educativo, acompañar a los estudiantes durante sus estudios de manera permanente y contribuir en la formación de competencias, es necesario implementar día a día nuevas y variadas formas de enseñar para lo cual es necesario realizar cambios permanentes a nivel docente, institucional y del sistema educativo en general.

Para finalizar se resalta la necesidad de implementar estrategias pedagógicas para facilitar la enseñanza de la educación física generando en la comunidad confianza sobre la experiencia laboral que se va adquiriendo con el tiempo, al desarrollar saberes para así gestionar proyectos comunitarios y obtener resultados óptimos, logrando suplir las necesidades y problemáticas que hacen parte de los diferentes contextos sociales, en colegios, hospitales, fundaciones, escuelas deportivas, entre otros. (Bernate, Fonseca \& Castillo, 2019)

\section{Conclusiones}

Se evidencia como prioridad el impacto social a nivel nacional e internacional con relación al aprendizaje, la satisfacción personal y el desempeño profesional, por medio de las intervenciones sociales que se imparten en las buenas habilidades desarrolladas de los docentes. El programa de 
licenciatura en educación física se considera como una oportunidad en la universidad, porque apoya los proyectos sociales para incentivar a las personas en aprender y desempeñar la educación física como parte de la cultura, los estudiantes implementan la responsabilidad social como eje integrador en sus funciones laborales y se incentiva el fortalecimiento de las habilidades sociales primero entre el alumno y el docente y después se imparte y socializa en la comunidad.

Finalmente, aunque el estudiante atañe un buen nivel en el aprendizaje y el desempeño profesional por parte del programa, también se demuestra que la satisfacción personal tiene en cuenta los alcances del conocimiento en función de suplir las problemáticas sociales desde el comienzo de la formación profesional. En efecto, se busca que el estudiante este en capacidad de dar respuesta a diversas situaciones laborales y tenga aptitud frente a otros estudiantes y próximos profesionales.

Por último, el programa académico evaluado aporta significativamente a la formación integral de los educadores físicos, en cuanto a habilidades profesionales propias del área específica y desarrolla competencias sociales, en pro de innovar continuamente la capacitación docente en educación superior.

\section{Referencias}

Álvarez Botello, J., Chaparro Salinas, E., \& Reyes Pérez, D. (2015). Estudio de la Satisfacción de los Estudiantes con los Servicios Educativos Brindados por Instituciones de Educación Superior del Valle de Toluca. REICE. Revista Iberoamericana Sobre Calidad, Eficacia Y Cambio En Educación, 13(2). Recuperado de https:// revistas.uam.es/index.php/reice/article/view/2788/3003

Arciniegas Ortiz, Jaime Alfonso, \& Mejías Acosta, Agustín Alexander. (2017). Percepción de la calidad de los servicios prestados por la Universidad Militar Nueva Granada con base en la escala Servqualing, con análisis factorial y análisis de regresión múltiple. Comuni@cción, 8(1), 26-36. Recuperado de http://www.scielo.org.pe/ scielo.php?script=sci_arttext\&amp;pid=S221971682017000100003

Bernate, J., Bejarano, B., \& Cardozo, D. (2020). Cotejo de las competencias ciudadanas en estudiantes de Licenciatura en Educación Física. Mendive. Revista de Educación, 18(3), 647-660. Recuperado de http://mendive.upr.edu.cu/ index.php/MendiveUPR/article/view/2049

Bernate, J. Fonseca, I. Betancourt, M. García, F. \& Sabogal, H. (2019) Competencias ciudadanas en la educación física escolar. Revista Acción Motriz, Asociación Científico Cultural en Actividad Física y Deporte (ACCAFIDE) Las Palmas de Gran Canaria, 23(2), 90- 99. Recuperado de: http://www.accionmotriz.com/documentos/revistas/ articulos/23_12.pdf

Bernate, J., Fonseca, I., \& Betancourt, M. (2019). Impacto de la actividad física y la práctica deportiva en el contexto social de la educación superior (Impact of physical activity and sports practice in the social context of higher education). Retos, 37(37), 742-747. Recuperado de https://recyt.fecyt.es/ index.php/retos/article/view/67875

Bernate, J., Fonseca, I., \& Castillo, E. (2019) Impacto social del deporte y la actividad física en el ámbito escolar. ATHLOS
Revista Internacional de Ciencias Sociales de la Actividad Física, el Juego y el Deporte International Journal of Social Sciences of Physical Activity, Game and Sport, 16(8), 78-97. Recuperado de: https://dialnet.unirioja.es/servlet/ articulo?codigo $=6837390$

Bernate, J., Fonseca, I. \& Urrea, P. (2019). Impacto en los procesos de la internacionalización y transnacionalización en la educación física superior UNIMINUTO. EmaìsF: Revista Digital de Educación Física, (61) 11-23. Disponible en: https://dialnet.unirioja.es/servlet/articulo?codigo=7133734

Bernate, J., Fonseca, I., Betancourt, M., \& Romero, E. (2020). Análisis de las competencias ciudadanas en estudiantes de Licenciatura en Educación Física. PODIUM - Revista de Ciencia y Tecnología en la Cultura Física, 15(2), 202 220. Recuperado de http://podium.upr.edu.cu/index.php/ podium/article/view/918

Bernate, J., Fonseca, I., Urrea, P., \& Amaya, G. (2019). La educación física y el deporte social para la paz y el posconflicto en Colombia. EmaìsF: revista digital de educación física, (61), 33-47. Recuperado de: https://dialnet.unirioja.es/servlet/ articulo? codigo $=7133736$

Betancourt, M., Bernate, J., Fonseca, I., \& Rodríguez, L. (2020). Revisión documental de estrategias pedagógicas utilizadas en el área de la educación física, para fortalecer las competencias ciudadanas (Documentary review of pedagogical strategies used in the area of physical education to strengthen citizen competenci. Retos, 38(38), 845851.Recuperado de https://recyt.fecyt.es/index.php/retos/ article/ view/74918/49523

Callado, C. (2015). Aprendizaje cooperativo en Educación Física: estado de la cuestión y propuesta de intervención (Cooperative learning in Physical Education: the state of the question and intervention proposal). Retos, 0(28), 234-239. Recuperado de https://recyt.fecyt.es/index.php/ retos/article/ view/35533/19669

Campos Mesa, M. C., Romero Granados, S., \& González Campos, G. (2015). La situación laboral del egresado en Magisterio de Educación Física: garantía de calidad (The labour situation of the graduates in Teaching in Physical Education: guarantee of quality). Retos, (17), 111-114. Recuperado de https:// recyt.fecyt.es/index.php/retos/article/ view/34688

Castrillón, E. P., \& Giraldo, S. A. (2016). Metodología para caracterización y estudio de impacto en el medio de egresados de instituciones de educación superior. Revista Interamericana de Investigación, Educación y

Pedagogía, RIIEP, 9(2). Disponible en: https:// revistas.usantotomas.edu.co/index.php/riiep/article/view/ 3616/3524

Castro Sandúa, M., Gómez González, A., \& Macazaga López, A. (2015). Aprendizaje dialógico y grupos interactivos en educación física (Dialogic learning and interactive groups in physical education). Retos, 0(25), 174- 179. Recuperado de: https://recyt.fecyt.es/index.php/retos/article/view/ 34508/ 18638

Clemenza, Caterina, \& Ferrer, Juliana, \& Pelekais, Cira (2005). La calidad como elemento competitivo en las Universidades. Revista de Artes y Humanidades UNICA, 6(14), 55-83. [Fecha de Consulta 9 de Junio de 2020]. ISSN: 1317-102X. Disponible en: https://www.redalyc.org/ articulo.oa?id=1701/ 170118766004

Clerici, R., \& Da Re, L. (2019). Evaluación de la eficacia de un programa de tutoría formativa. Revista de investigación educativa, 37(1), 39-56. Recuperado de https:// revistas.um.es/rie/article/view/322331/254291 
Cruz, J. C. O., Toro, A. O., \& Duarte, V. V. (2009). ¿Calidad de la educación o educación de calidad? Una preocupación más allá del mercado. Revista Iberoamericana de educación, (51), 161-181. Recuperado de: https://www.redalyc.org/pdf/ 800/80012433010.pdf

Fernández-Rio, J., \& Méndez-Giménez, A. (2015). El Aprendizaje Cooperativo: Modelo Pedagógico para Educación Física (Cooperative learning: Pedagogical Model for Physical Education). Retos, O(29), 201- 206. Recuperado de https:// recyt.fecyt.es/index.php/retos/article/view/ 38721/25521

Fonseca, I., Bernate, J., Betancourt, M., Barón, B., \& Cobo, J. (2019). Developing Social Responsibility in University Students. In Proceedings of the 2019 11th International Conference on Education Technology and Computers (ICETC 2019). Association for Computing Machinery, New York, NY, USA, 215-218. Doi: https://doi.org/10.1145/ 3369255.3369275

Gallardo-Fuentes, F., \& Carter Thuillier, B. (2015). La evaluación formativa y compartida durante el prácticum en la formación inicial del profesorado: Análisis de un caso en Chile (Formative and Shared Assessment during the prácticum on the Preservice Teacher Education: a case analysis in Chile). Retos, O(29), 258-263. Recuperado de https://recyt.fecyt.es/ index.php/retos/article/view/43550/25491

García Martínez, S., Sánchez Blanco, P., \& Ferriz Valero, A. (2020). Metodologías cooperativas versus competitivas: efectos sobre la motivación en alumnado de EF (Cooperative versus competitive methodologies: effects on motivation in $\mathrm{PE}$ students). Retos, (39), 65-70. Recuperado de https:// recyt.fecyt.es/index.php/retos/article/view/78279

Gómez, U., Quiroz, L., \& Zambrano, J. (2016). Criterios para la evaluación del impacto académico de programas de maestría en la modalidad semipresencial.Didasc@ lia: Didáctica y Educación, 7(5), 85-96. Recuperado de: https:// dialnet.unirioja.es/servlet/articulo? codigo $=5911162$

Gracia Rojas, L. C. (2018). Evaluación de impacto en los egresados del programa de especialización en evaluación de y gerencia de proyectos de Bucaramanga en las cohortes de los últimos cinco aã o (Doctoral dissertation, Universidad Industrial de Santander, Escuela De Estudios Industriales Y Empresariales). Disponible en: http://noesis.uis.edu.co/ handle/123456789/10716

Hernández, C., López, M., \& Olmedo, E. (2015). Evaluación de la satisfacción de estudiantes universitarios de nuevo ingreso con el programa E.S.C.L.O.S.A.: experiencia de un programa de orientación. REOP - Revista Española de Orientación y Psicopedagogía, 26(2), 123-135.doi:https://doi.org/10.5944/ reop.vol.26.num.2.2015.15219

Hernández-Pina, F. y Maquilón, J.J. (2010). Introducción a los diseños de investigación educativa. En S. Nieto (Ed.), Principios, métodos y técnicas esenciales para la investigación educativa (pp.109-126). Madrid: Dykinson. Recuperado de: https://dialnet.unirioja.es/servlet/ articulo? codigo $=3416704$

Juliao Vargas, C. G (2011). El enfoque praxeológico. Recuperado de: https://repository.uniminuto.edu/handle/10656/1446

Maya Guerra, José Ignacio, \& Herrera Herrera, María Eugenia (2011). Caracterización e impacto de los graduados de la Católica del Norte Fundación Universitaria. Revista Virtual Universidad Católica del Norte, (34), 78-112. Recuperado de:

https://revistavirtual.ucn.edu.co/index.php/RevistaUCN/article/ view/331/633

Paez, J., \& Hurtado Almonacid, J. (2018). Formación inicial docente en profesores de educación física. Levantamiento de competencias específicas a partir de las necesidades del medio educativo (Initial teacher training in physical education teachers. Determining specific skills based on contextu. Retos, (35), 61-66. Recuperado a partir de https://recyt.fecyt.es/ index.php/retos/article/view/61887

Pérez-Albéniz, A., Pascual, A. I., Navarro, M. C., \& Lucas-Molina, B. (2015). Más allá del conocimiento. El impacto de un programa educativo universitario para mayores. Aula abierta, 43(1), 54-60. https://www.sciencedirect.com/science/ article/pii/S0210277314000237

Roca Cruz, A., Cabello Manrique, D., Gonzalez, J., \& CourelIbáñez, J. (2017). Estudio de satisfacción de los asistentes a la Universiada de Invierno Granada 2015 (Study on attendees' satisfaction at the Winter University Games of Granada 2015). Retos, (33), 247-251. Recuperado a partir de https:// recyt.fecyt.es/index.php/retos/article/view/58614

Rojas, M., Bernate, J., Fonseca, I., \& Betancourt, M. (2019) El método praxeologico como herramienta pedagógica de aprendizaje motriz. ATHLOS Revista Internacional de Ciencias Sociales de la Actividad Física, el Juego y el Deporte International Journal of Social Sciences of Physical Activity, Game and Sport, (18)3, 12-35. Recuperado de: https://dialnet.unirioja.es/servlet/ articulo? codigo $=7086787$

Ryan, R., \& Deci, E. L. (2000). La Teoría de la Autodeterminación y la Facilitación de la Motivación Intrínseca, el Desarrollo Social, y el Bienestar. American Psychologist, 55(1), 68-78. Disponible en https://selfdeterminationtheory.org/SDT/ documents/2000_RyanDeci_SpanishAmPsych.pdf

Serra-Olivares, J., Muñoz Valverde, C., Cejudo Armero, C., \&amp; Gil Madrona, P. (2016). Estilos de aprendizaje y rendimiento académico de universitarios de Educación Física chilenos (Learning styles and academic performance of Chilean Physical Education university students). Retos, O(32), 62-67. Recuperado de https://recyt.fecyt.es/index.php/retos/article/ view/51919/33574

Surdez, E. G, Sandoval, Mdel C.y Lamoyi, C.L.(2018). Satisfacción estudiantil en la valoración de la calidad educativa universitaria. Educación y Educadores, 21(1), 9-26. Doi: https://doi.org/10.5294/edu.2018.21.1.1

UNESCO (1998) La Educación superior en el siglo XXI, visión y acción: informe final. Recuperado de: https:// unesdoc.unesco.org/ark:/48223/pf0000116345_spa

Urrea, P., Bernate, J., \& Fonseca, I. (2019) Docentes formadores para la paz, desde las practicas pedagógicas. Revista de Alesde Curitiba (10)1, 92-100. DOI: http://dx.doi.org/10.5380/ jlasss.v10i1.67594

Urrea, P., Bernate, J. A., Fonseca, I. y Martínez, A. (2018). Impacto social de los graduados de la Licenciatura en Educación Física, Recreación y Deporte UNIMINUTO. Educación Física y Ciencia, 20 (2), e050. https://doi.org/10.24215/1514$0105 \mathrm{e} 050$

Zueck Enríquez, M., Ramírez García, A., Rodríguez Villalobos, J., \& Irigoyen Gutiérrez, H. (2019). Satisfacción en las clases de Educación Física y la intencionalidad de ser activo en niños del nivel de primaria (Satisfaction in the Physical Education classroom and intention to be physically active in Primary school children). Retos, 37(37), 33-40. Recuperado a partir de https://recyt.fecyt.es/index.php/retos/article/view/69027 